\title{
Severe weight loss during preoperative chemoradiotherapy compromises survival outcome for patients with locally advanced rectal cancer
}

\author{
Junzhong Lin ${ }^{1} \cdot$ Jianhong Peng ${ }^{1} \cdot$ Aiham Qdaisat ${ }^{2} \cdot$ Liren $\mathbf{L i}^{1} \cdot$ Gong Chen $^{1}$ \\ Zhenhai $\mathrm{Lu}^{1} \cdot$ Xiaojun $\mathrm{Wu}^{1} \cdot$ Yuanhong Gao ${ }^{3} \cdot$ Zhifan Zeng $^{3} \cdot$ Peirong Ding ${ }^{1}$. \\ Zhizhong Pan ${ }^{1}$
}

Received: 16 August 2016 / Accepted: 22 August 2016 / Published online: 9 September 2016

(C) The Author(s) 2016. This article is published with open access at Springerlink.com

\begin{abstract}
Purpose In addition to tumor factors, poor nutritional status before and during anti-tumor treatment might compromise prognosis in several types of cancer. This study was done to determine the impact of weight loss during preoperative chemoradiotherapy (CRT) on the survival outcome of patients with locally advanced rectal cancer (LARC).

Methods The retrospective study examined consecutive patients with LARC who underwent preoperative CRT followed by radical resection in a single institute, between 2003 and 2013. Correlation of proportional body mass index (BMI) change after preoperative CRT and patient's demographics, tumor characteristics, treatment parameters, CRT-related toxicity, disease-free survival (DFS) and overall survival (OS) were investigated.

Results A total of 364 patients were enrolled, and BMI loss was found in 243 patients $(66.2 \%)$ after preoperative CRT.
\end{abstract}

Junzhong Lin, Jianhong Peng and Aiham Qdaisat have contributed equally to this work.

Peirong Ding

dingpr@sysucc.org.cn

$\triangle$ Zhizhong Pan

panzhzh@sysucc.org.cn

1 Department of Colorectal Surgery, Sun Yat-sen University Cancer Center, State Key Laboratory of Oncology in South China, Collaborative Innovation Center for Cancer Medicine, 651 Dongfeng Road East, Guangzhou 510060,

People's Republic of China

2 Department of Emergency Medicine, The University of Texas MD Anderson Cancer Center, Houston, TX 77030, USA

3 Department of Radiation Oncology, Sun Yat-sen University Cancer Center, State Key Laboratory of Oncology in South China, Collaborative Innovation Center for Cancer Medicine, Guangzhou 510060, People's Republic of China
Severe weight loss (SWL) was defined as BMI loss $\geq 7 \%$. Thirty-nine $(10.7 \%)$ cases were enrolled in SWL cohort and found to have higher incidence of diarrhea $(P=0.033)$, renal disorder $(P=0.033)$ and grade $3-4$ radiation proctitis $(P=0.041)$. Although no significant difference was found in 3-year DFS, patients in SWL cohort showed significantly worse 3-year OS rate (71.8 vs $88.0 \%, P=0.030$ ) than the others. In univariate analysis, BMI loss $\geq 7 \%$, completed dose of preoperative chemotherapy, pathologic $\mathrm{T}$ and $\mathrm{N}$ stages were correlated with OS (all $P<0.05$ ). In multivariable analysis, BMI loss $\geq 7 \%$ (HR 1.984; $95 \%$ CI 1.061-3.709; $P=0.032$ ) remained the independent prognostic factor for OS.

Conclusions Our results demonstrate that SWL during preoperative CRT indeed compromises survival outcome in patients with LARC. Routine nutritional monitoring and nutritional support during preoperative CRT are suggested as the integral part of the multidisciplinary approach for these patients.

Keywords Weight loss - Preoperative chemoradiotherapy · Survival outcome $\cdot$ Rectal cancer

\section{Introduction}

Preoperative chemoradiotherapy (CRT) followed by total mesorectal excision (TME) has been demonstrated as the effective treatment pattern for patients with locally advanced rectal cancer (LARC) (Bosset et al. 2006; Sauer et al. 2004). Although most of these patients could achieve improvement of local disease control and sphincter preservation, a proportion of patients failed to benefit from preoperative CRT (Diaz-Gonzalez et al. 2006; Kong et al. 2012). Therefore, it is necessary to identify risk factors associated with prognosis and individualize the therapy based on the 
oncologic outcome analysis. In addition to tumor factors, many studies supported the idea that poor nutritional status before and during anti-tumor treatment could compromise prognosis in some types of cancer (Cooper et al. 2015; Iseki et al. 2015; Sakurai et al. 2016).

Body mass index (BMI), as a crucial indicator in the assessment of nutritional status, is easy to measure with low cost and may be associated with mortality of patients suffering from cancer (Parr et al. 2010; Reeves et al. 2007). Recently, lower preoperative BMI has been identified as a risk factor for poor prognosis of rectal cancer (Adachi et al. 2015; Uratani et al. 2015). Since malnutrition commonly occurred during chemotherapy and radiotherapy, the changes in nutritional status during treatment should be taken into consideration (Gudny Geirsdottir and Thorsdottir 2008; Koom et al. 2012). To date, data remain limited about the impact of weight loss during neoadjuvant treatment on long-term survival for LARC patients. We hypothesized that severe weight loss during preoperative CRT resulted in a worse patient's survival. Herein, we conducted this retrospective study to investigate the association of weight loss during preoperative CRT and the survival outcome in patients with LARC.

\section{Patients and methods}

\section{Patient selection}

Patients with LARC undergoing preoperative CRT followed by total mesorectal excision at Sun Yat-sen University Cancer Center during March 2003-April 2013 were retrospectively identified. Enrolled patients met the following inclusion criteria: (1) histologically confirmed rectal adenocarcinoma; (2) T3-4 or N+ disease initially; and (3) radical resection for rectal tumor. The exclusion criteria were as follow: (1) missing height and weight records of pre- or post-CRT; (2) metastatic disease before or during preoperative treatment; and (3) other active malignancy (except for basal cell carcinoma of the skin). Patient demographic, preoperative and postoperative treatment, CRT toxic reaction and follow-up results were reviewed in detail from the medical records and the follow-up system. Current study was undertaken in accordance with the ethical standards of the World Medical Association Declaration of Helsinki. A waiver of informed consent was requested, and the approval was obtained from independent ethics committees at Sun Yat-Sen University Cancer Center.

\section{Treatments}

All patients underwent preoperative CRT in the parallel pattern. Irradiation was scheduled in total dose of 46.0 50.40 Gy to the pelvic area, delivered in fractions of 1.8 or 2.0 Gy daily on five consecutive days per week during 6 weeks. Concurrent chemotherapy regimen was administered as follow: XELOX regimen (oxaliplatin $130 \mathrm{mg} / \mathrm{m}^{2}$ on day 1 and capecitabine $1000 \mathrm{mg} / \mathrm{m}^{2}$ twice daily on days 1-14 were given for 3 week-cycle); or FOLFOX6 regimen (oxaliplatin $85 \mathrm{mg} / \mathrm{m}^{2}$ and leucovorin $400 \mathrm{mg} / \mathrm{m} 2$ were administered on day 1, 5-FU was injected intravenously $400 \mathrm{mg} / \mathrm{m}^{2}$ on day 1 and then administered $2400 \mathrm{mg} / \mathrm{m}^{2}$ by intravenous infusion on days 1 and 2 for 2 week-cycle); or capecitabine only (capecitabine $825 \mathrm{mg} / \mathrm{m}^{2}$ was given twice daily during radiotherapy without weekend breaks). All patients underwent radical TME resection 6-8 weeks after the end of the preoperative radiotherapy. The recommended oxaliplatin-based adjuvant chemotherapy started 3-6 weeks after surgery.

\section{Measurements}

Height and weight of overall patients were measured 1 day before CRT and surgery. BMI was calculated as weight $(\mathrm{kg}) /$ height square $\left(\mathrm{m}^{2}\right)$, and its relative change was generated. BMI level was defined according to the Chinese standard (Zeng et al. 2014): higher BMI (overweight or obese), $>24 \mathrm{~kg} / \mathrm{m}^{2}$; normal BMI, $18.5-24 \mathrm{~kg} / \mathrm{m}^{2}$; and lower BMI (underweight), $\leq 18.5 \mathrm{~kg} / \mathrm{m}^{2}$. Pretreatment staging was determined by endorectal ultrasound (EUS), chest and abdominopelvic computed tomography scanning (CT) and/ or pelvic magnetic resonance imaging (MRI). Acute toxicities during CRT were graded according to the National Cancer Institute Common Toxicity Criteria (NCI CTC) version 4.0. Pathological assessments of the resected specimens were confirmed according to tumor-node-metastasis (TNM) classification by two independent pathologists. Pathologic complete response (pCR) was defined as follow: the absence of viable tumor cells with only fibrotic masses or a cellular mucin pools present in area of primary tumor and lymph nodes (Mandard et al. 1994).

\section{Follow-up}

The primary endpoint was overall survival (OS), and the secondary endpoints were disease-free survival (DFS). OS was defined as time length from operation to death from any cause, while DFS was defined as the interval from tumor resection to disease recurrence. All patients were observed through subsequent visit every 3 months for 2 years and then semiannually until 3 years after surgery. Evaluation included clinical examination, carcinoembryonic antigen (CEA) level, abdominal ultrasonography and chest radiograph. A chest computed tomography (CT), abdominal/pelvic magnetic resonance (MRI) and colonoscopy were performed annually. The last follow-up visit occurred in March 2016. 


\section{Statistical analysis}

Clinical data were analyzed using Statistical Package for the Social Sciences (SPSS, version 17.0, Chicago, IL). Continuous variables were presented as mean (standard deviation, SD) or median (range), which were compared using Student $t$ test or Mann-Whitney test. Categorical variables were given as percentage and then compared by applying Chi-square test or Fisher's exact between groups, when appropriate. Receiver operating characteristic curve (ROC) analysis was used to determine the cutoff point of BMI change according to OS. The OS and DFS rates were estimated by the Kaplan-Meier method, and differences between the two groups were assessed by the log-rank test. Variables proved statistical significance in univariate Cox models were further assessed by multivariate Cox models. The multivariate Cox proportional hazards model was used to identify independent prognostic factors for OS, and the hazard ratios (HRs) and confidence intervals (CIs) were calculated. All statistical tests were two-sided, and the significant level was set at 0.05 .

\section{Results}

\section{Patient characteristics and cutoff point for weight loss}

A total of 364 patients were enrolled. The characteristics of BMI and body weight for these patients are shown in Table 1. Initially, $40(11 \%)$ patients were underweight, while $108(29.7 \%)$ were overweight. BMI and weight significantly decreased during CRT, especially for overweight patients, and BMI loss was found in 243 patients $(66.2 \%)$.

The receiver operating characteristic (ROC) analysis indicated the optimal cutoff value of BMI change for OS was $7 \%(P=0.031$, Fig. 1$)$. Therefore, severe weight loss (SWL) during CRT was defined as BMI loss $\geq 7 \%$. As a result, $39(10.7 \%)$ patients with BMI loss $\geq 7 \%$ were included in the SWL cohort, while the others with BMI loss $<7 \%$, no BMI loss or even BMI gain during CRT were grouped into non-severe weight loss (non-SWL) cohort.

Patients in SWL cohort seemed to be older than those in non-SWL cohort (62 vs 55 years, $P=0.007$ ). Despite of age, clinical characteristics of the two groups including gender distribution, baseline BMI, distance of inferior tumor margin from the anal verge (DAV), tumor size and pretreatment TNM stage were comparable (Table 2). In addition, there was no significant difference in the pathological outcome after CRT and surgical resection between the two groups.

\section{Treatment characteristics and chemoradiotherapy toxicity}

In regard to treatment parameters of preoperative CRT and surgery, no significant difference was found between SWL and non-SWL cohorts, as presented in Table 3. However, a smaller proportion in SWL cohort received postoperative chemotherapy (69.2 vs $83.4 \%, P=0.030$ ), and a shorter duration of chemotherapy was delivered (10.5 vs 12 weeks, $P=0.019)$, compared to that in non-SWL cohort.

Treatment-related adverse events during preoperative CRT are shown in Table 4. Despite similar incidence in the majority of the adverse events, patients with BMI loss $\geq 7 \%$ had significantly higher occurrence rate of diarrhea (48.7 vs $31.4 \%, P=0.033$ ), renal disorder (5.1 vs $0.6 \%$, $P=0.033)$ and severe (grade 3-4) radiation proctitis (7.7 vs $1.8 \%, P=0.041)$.

\section{Survival outcomes and severe weight loss}

A total of $83(22.8 \%)$ patients ultimately developed postoperative recurrences during a median 47.8-month followup (range 4-130 months). The 3-year DFS and OS rate

Table 1 Body mass index and body weight change during preoperative chemoradiotherapy with patients of locally advanced rectal cancer

\begin{tabular}{|c|c|c|c|c|c|c|}
\hline Factors & $N(\%)$ & Pre-CRT & Post-CRT & Relative change (median, range) & Variation $(95 \% \mathrm{CI})$ & $P$ value \\
\hline \multicolumn{7}{|c|}{ Pre-CRT BMI (mean $\left.\pm \mathrm{SD}, \mathrm{kg} / \mathrm{m}^{2}\right)$} \\
\hline Total & 364 & $22.4 \pm 3.1$ & $22.3 \pm 3.1$ & $-0.71 \%(-25.97$ to $30.91 \%)$ & $-0.163(-0.295$ to -0.030$)$ & 0.02 \\
\hline$\leq 18.5$ & $40(11.0)$ & $17.2 \pm 1.3$ & $17.4 \pm 1.6$ & $0(-9.96$ to $22.08 \%)$ & $0.257(-0.143$ to 0.657$)$ & 0.20 \\
\hline $18.5-24$ & $216(59.3)$ & $21.6 \pm 1.5$ & $21.5 \pm 1.8$ & $0(-14.29$ to $30.91 \%)$ & -0.077 ( -0.240 to 0.086$)$ & 0.35 \\
\hline$>24$ & $108(29.7)$ & $26.1 \pm 1.7$ & $25.6 \pm 2.0$ & $-1.29 \%(-12.70$ to $25.97 \%)$ & $-0.489(-0.754$ to -0.225$)$ & $<0.001$ \\
\hline \multicolumn{7}{|c|}{ PreCRT body weight (mean $\pm \mathrm{SD}, \mathrm{kg}$ ) } \\
\hline Total & 364 & $60.5 \pm 9.7$ & $60.1 \pm 9.8$ & $-0.71 \%(-25.97$ to $30.91 \%)$ & $-0.418(-0.771$ to -0.056$)$ & 0.02 \\
\hline$\leq 50$ & $50(13.7)$ & $45.5 \pm 4.1$ & $45.7 \pm 5.1$ & $-1.10 \%(-11.46$ to $22.08 \%)$ & $0.224(-0.724$ to 1.172$)$ & 0.64 \\
\hline $50-70$ & $256(70.3)$ & $60.1 \pm 5.7$ & $59.7 \pm 6.2$ & $0(-17.07$ to $30.91 \%)$ & $-0.330(-0.731$ to 0.071$)$ & 0.11 \\
\hline$>70$ & $58(15.9)$ & $75.4 \pm 4.2$ & $74.1 \pm 5.8$ & $-1.32 \%(-25.97$ to $8.97 \%)$ & $-1.333(-2.458$ to -0.207$)$ & 0.02 \\
\hline
\end{tabular}

$B M I$ body mass index, $S D$ standard deviation, $C I$ confidence interval, $C R T$ chemoradiotherapy 
Fig. 1 Comparison of different cutoff points of BMI loss based on overall survival outcome by a area under the curve (AUC) and $\mathbf{b}$ the minimal $P$ value
A

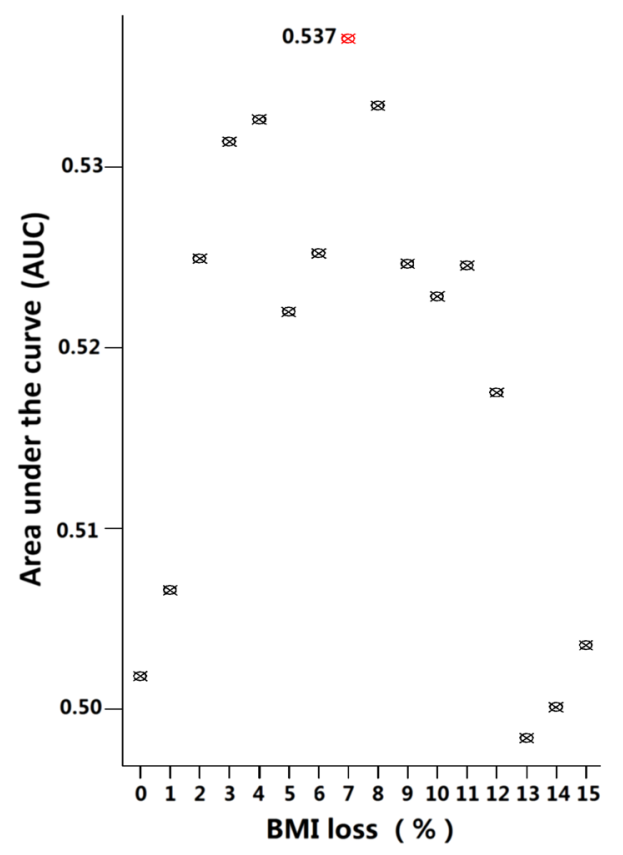

B

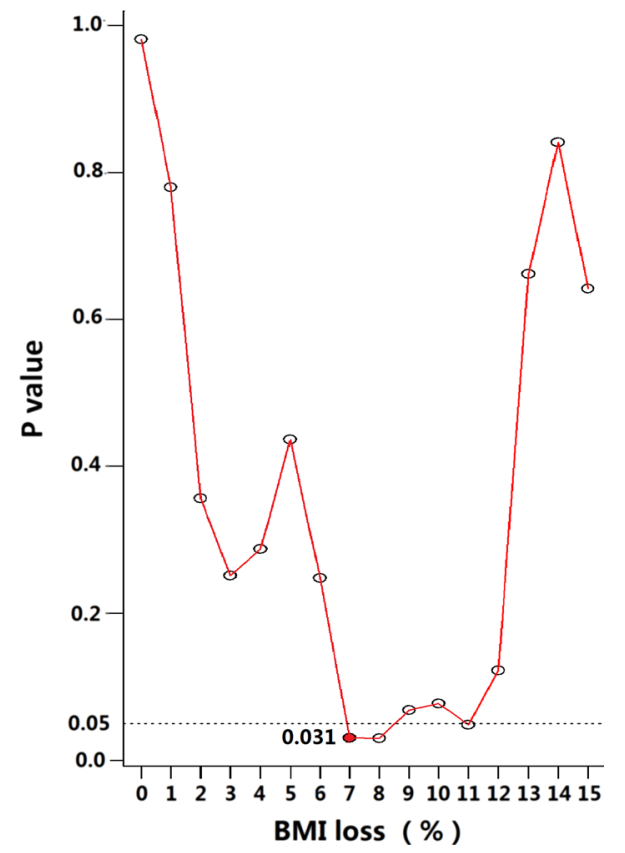

were 83.3 and $86.0 \%$, respectively. Among the baseline subgroups of underweight, normal BMI and overweight or obese patients, neither 3-year DFS rates (79.6 vs 83.7 vs $85.1 \%, P=0.770$; Fig. 2a) nor 3-year OS rates (89.7 vs 83.4 vs $90.5 \%, P=0.488$; Fig. 3a), showed any statistical difference. Similarly, no significant difference was found according to the post-CRT BMI (DFS: $P=0.820$ and OS: $P=0.305)$. Although no significant difference was found in the 3-year DFS (81.1 vs $83.7 \%, P=0.655$; Fig. $2 b$ ), patients in SWL cohort had significantly worse 3-year OS compared to non-SWL cohort (71.8 vs $88.0 \%, P=0.030$; Fig. 3b). As shown in Table 5, univariate analysis revealed that BMI loss $\geq 7 \%$ during preoperative CRT $(1.961 ; 95 \%$ CI $1.055-3.647 ; P=0.030$ ), completed dose of preoperative chemotherapy $\leq 50 \%$ and advanced pathologic $\mathrm{T}$ and $\mathrm{N}$ stages were associated with poor OS. The multivariate analysis subsequently demonstrated that all factors above including BMI loss $\geq 7 \%$ (HR 1.984; $95 \%$ CI 1.0613.709; $P=0.032$ ) were independent prognostic factors for OS.

\section{Discussion}

It is now clear that cancer survival is determined not only by tumor pathology but also by host-related factors, in particular, nutritional status and systemic inflammation (Bachmann et al. 2015; Read et al. 2006). Previous studies have addressed the impact of pretreatment BMI on rectal cancer patient's outcomes (Campbell et al. 2012; Hede et al.
2015). Moreover, it has been reported that severe weight loss after treatment increased the risk of cancer recurrence and mortality in endometrial cancer and triple-negative breast cancer (Bao et al. 2016; Matsuo et al. 2016). However, the correlation of weight loss during preoperative CRT and survival outcome of LARC patients is not yet established. In this study, we found that severe weight loss during preoperative CRT was the independent risk factor for 3-year OS in LARC patients, instead of the baseline or post-CRT BMI.

Several reasons could contribute to the association between weight loss and poor survival outcome. Of these, the most critical reason is the impact of nutrient on cancer development. Polyunsaturated fatty acids (PUFAs), as one series of essential fatty acids, have been confirmed of having anti-tumor effects by inducing tumor cell apoptosis and inhibiting cell proliferation (Song et al. 2011; Xu and Qian 2014). Weight loss during preoperative CRT is always accompanied by insufficient fatty acids intake, which impairs the therapy effects. On the other hand, the role of immuonutrition is recognized as an important factor in modulating cancer progression. Mounting evidence showed that certain dietary nutrients were associated with the development of different types of cancers either by enhancing the immune system or by exerting an immunosuppressive effect (Valdes-Ramos and Benitez-Arciniega 2007; Yaqoob and Calder 2007). Previous study revealed that once weight loss occurs during treatment, immune function might be impaired, especially cell-mediated immunity (Fontana et al. 2007). Another crucial factor for decreased 
Table 2 Clinical and pathological characteristics of patients with locally advanced rectal cancer

\begin{tabular}{|c|c|c|c|c|}
\hline Variables & $\begin{array}{l}\text { All patients } \\
n=364(\%)\end{array}$ & $\begin{array}{l}\text { BMI loss }<7 \% \\
n=325(\%)\end{array}$ & $\begin{array}{l}\text { BMI loss } \geq 7 \% \\
n=39(\%)\end{array}$ & $P$ value \\
\hline \multicolumn{5}{|l|}{ Clinical variables } \\
\hline Age, median (range) & $56(15-80)$ & $55(15-80)$ & $62(33-76)$ & 0.007 \\
\hline Gender (male: female) & $259: 115$ & 224:101 & $25: 14$ & 0.54 \\
\hline Mean preCRT BMI $\left(\mathrm{kg} / \mathrm{m}^{2}\right)$ & $22.5 \pm 3.1$ & $22.4 \pm 3.1$ & $22.6 \pm 2.9$ & 0.72 \\
\hline $\begin{array}{l}\text { Mean inferior tumor margin from } \\
\text { anal verge, } \mathrm{cm}(\mathrm{SD})\end{array}$ & $4.9 \pm 2.2$ & $4.9 \pm 2.1$ & $5.2 \pm 2.7$ & 0.35 \\
\hline cT stage & & & & 0.46 \\
\hline 3 & $216(59.3)$ & $195(60.0)$ & $21(53.8)$ & \\
\hline 4 & $148(40.7)$ & $130(40.0)$ & $18(46.2)$ & \\
\hline cTNM stage & & & & 0.12 \\
\hline II & $127(34.9)$ & $109(33.5)$ & $18(46.2)$ & \\
\hline III & $237(65.1)$ & $216(66.5)$ & $21(53.8)$ & \\
\hline \multicolumn{5}{|l|}{ Pathological variables } \\
\hline Mean size of tumor after CRT, cm (SD) & $3.0 \pm 1.6$ & $3.0 \pm 1.5$ & $3.4 \pm 1.9$ & 0.14 \\
\hline Tumor differentiation & & & & 0.90 \\
\hline Well and moderate & $283(77.7)$ & $253(77.8)$ & $30(76.9)$ & \\
\hline Poor & $81(22.3)$ & $72(22.2)$ & $9(23.1)$ & \\
\hline Median number of lymph nodes examined (range) & $6(0-37)$ & $6(0-37)$ & $6(0-21)$ & 0.99 \\
\hline Median number of positive lymph nodes (range) & $0(0-12)$ & $0(0-12)$ & $0(0-4)$ & 0.42 \\
\hline ypT stage & & & & 0.42 \\
\hline $0-2$ & $181(49.7)$ & $164(50.5)$ & $17(43.6)$ & \\
\hline $3-4$ & $183(50.3)$ & $161(49.5)$ & $22(56.4)$ & \\
\hline ypN stage & & & & 0.50 \\
\hline 0 & $283(77.7)$ & $251(77.2)$ & $32(82.1)$ & \\
\hline $1-2$ & $81(22.3)$ & $74(22.8)$ & $7(17.9)$ & \\
\hline ypTNM stage & & & & 0.87 \\
\hline 0 & $85(23.4)$ & $76(23.4)$ & $9(23.1)$ & \\
\hline I & $77(21.2)$ & $69(21.2)$ & $8(20.5)$ & \\
\hline II & $121(33.2)$ & $106(32.6)$ & $15(38.5)$ & \\
\hline III & $81(22.2)$ & $74(22.8)$ & 7 (17.9) & \\
\hline Achievement of pCR & & & & 0.97 \\
\hline Yes & $85(23.4)$ & $76(23.4)$ & $9(23.1)$ & \\
\hline No & $279(76.6)$ & 249 (76.6) & $30(76.9)$ & \\
\hline
\end{tabular}

$B M I$ body mass index, SD standard deviation, $c T N M$ stage clinical tumor-node-metastasis classification, $c T$ stage clinical tumor stage, ypT stage pathologic tumor stage after chemoradiotherapy, ypN stage pathologic node stage after chemoradiotherapy, ypTNM stage pathologic tumornode-metastasis classification after chemoradiotherapy, $p C R$ pathologic complete response

survival outcome in patients with SWL during CRT is possibly the different compliance of postoperative chemotherapy. In our study, a significant lower proportion of patients in SWL cohort received postoperative chemotherapy, and these patients even had a shorter duration of chemotherapy, suggesting that the malnutrition status during CRT could compromise postoperative treatment compliance, which might influence the survival outcome.

As for the cause of weight loss, CRT-related toxicity may account for that. Our study showed that patients with
BMI loss $\geq 7 \%$ experienced higher incidence of diarrhea, renal disorder and grade 3-4 radiation proctitis during preoperative CRT. Similarly, Arrieta et al. (2010) noted that chemotherapy-induced toxicity in non-small cell lung cancer (NSCLC) patients treated with paclitaxel and cisplatin was associated with malnutrition and hypoalbuminemia. Recently, many studies found that patients with malnutrition or weight loss $\geq 5 \%$ during chemotherapy had higher risk of severe gastrointestinal toxicity, thus suggesting that evaluation of nutritional status was helpful in identifying 
Table 3 Treatment characteristics for patients with locally advanced rectal cancer

\begin{tabular}{|c|c|c|c|}
\hline Variables & $\begin{array}{l}\text { BMI loss }<7 \% \\
n=325(\%)\end{array}$ & $\begin{array}{l}\text { BMI loss } \geq 7 \% \\
n=39(\%)\end{array}$ & $P$ value \\
\hline \multicolumn{4}{|l|}{ Preoperative chemoradiotherapy } \\
\hline Chemotherapy regimen & & & 0.25 \\
\hline XELOX & $273(84.0)$ & $32(82.1)$ & \\
\hline FOLFOX & $38(11.7)$ & $7(17.9)$ & \\
\hline Capecitabine & $14(4.3)$ & 0 & \\
\hline Duration of chemotherapy, cycles (median, range) & $2(1-4)$ & $2(1-4)$ & 0.27 \\
\hline Completion of chemotherapy & $258(79.4)$ & $28(71.8)$ & 0.26 \\
\hline Completed dose of chemotherapy $>50 \%$ & $298(91.7)$ & $37(94.9)$ & 0.49 \\
\hline Dose of radiotherapy, Gy (range) & $46(30-50)$ & $46(30-50)$ & 0.32 \\
\hline Completion of radiotherapy & $317(97.5)$ & $37(94.9)$ & 0.35 \\
\hline \multicolumn{4}{|l|}{ Surgical operation } \\
\hline $\begin{array}{l}\text { Interval from the completion of radiation } \\
\text { to surgery, weeks (range) }\end{array}$ & $6.8(1-12)$ & $7(4-11)$ & 0.99 \\
\hline Surgical procedure & & & 0.66 \\
\hline Anterior resection & $187(57.5)$ & $21(53.8)$ & \\
\hline Abdominal perineal resection & $138(42.5)$ & $18(46.2)$ & \\
\hline \multicolumn{4}{|l|}{ Postoperative treatment } \\
\hline Adjuvant chemotherapy & & & 0.030 \\
\hline Yes & $271(83.4)$ & $27(69.2)$ & \\
\hline No & $54(16.6)$ & $12(30.8)$ & \\
\hline $\begin{array}{l}\text { Duration of postoperative chemotherapy, } \\
\text { weeks (median, range) }\end{array}$ & $12(0-18)$ & $10.5(0-18)$ & 0.019 \\
\hline
\end{tabular}

$B M I$ body mass index, $C R T$ chemoradiotherapy patients at higher risk of severe gastrointestinal toxicity (Arrieta et al. 2015; Sanchez-Lara et al. 2013). It is worthy to note that nutritional changes during neoadjuvant treatment should be taken into consideration when adjusting chemotherapy and radiotherapy doses, in order to minimize the CRT-related toxicity in LARC patients.

Interestingly, our data revealed that overweight or obese LARC patients were more likely to experience more weight loss during preoperative CRT (shown in Table 1). Similarly, a Sweden nationwide cohort study has demonstrated that overweight patients with esophageal cancer were of a higher risk of malnutrition after esophagostomy (Martin and Lagergren 2009). The possible reason might be attributed to the higher administrated dose of chemotherapy and worse tolerability of CRT toxicity in those patients. In fact, a recent study showed that the risk of dose limiting toxicity was obviously increased in sarcopenic obese esophageal cancer patients (OR 5.54; $95 \%$ CI 1.12-27.44, $P=0.04$ ) (Anandavadivelan et al. 2016). Thus, malnutrition should be alerted during adjuvant treatment in overweight or obese LARC patients.

As we have mentioned above, BMI loss during preoperative CRT, which could compromise long-term OS, was common and occurred in $66.2 \%$ of LARC patients. Therefore, it is necessary to pay more attention to the re-evaluation of nutritional status and nutritional balance in the process of anti-cancer therapy. Recently, a prospective study has demonstrated that nutrition support combined with treatment could prolong survival in advanced gastric cancer patients with malnutrition (median survival: 14.3 vs 9.6 months, $P=0.001$ ) (Qiu et al. 2015). Another small volume randomized trial also confirmed the effectiveness of individualized nutritional counseling and early nutritional education during radiotherapy in improving longterm prognosis in colorectal cancer (Ravasco et al. 2012). Furthermore, feasible nutrition support during neoadjuvant chemotherapy helped to reduce the incidence of chemotherapy-related toxicities (Miyata et al. 2012). Thereby, nutritional intervention should be an early approach and targeted for each LARC patient who undergo preoperative CRT, including personalized dietary counseling and artificial nutrition support, based on spontaneous food intake, toxicity tolerance and nutritional status.

Several potential limitations should be acknowledged. First of all, weight loss does not totally represent malnutrition status. Other reported methods for the evaluation include descending change in serum albumin and hemoglobin level during treatment, as well as the scored Patient Generated Subjective Global Assessment (PG-SGA) (Das et al. 2014). Nevertheless, weight loss is closely related 
Table 4 Treatment-related adverse events during preoperative chemoradiotherapy in patients with locally advanced rectal cancer

\begin{tabular}{|c|c|c|c|c|c|c|c|}
\hline Toxicity & $\begin{array}{l}\text { BMI loss }<7 \% \\
n=235(\%)\end{array}$ & $\begin{array}{l}\text { BMI loss } \geq 7 \% \\
n=39(\%)\end{array}$ & $P$ value & Toxicity & $\begin{array}{l}\text { BMI loss }<7 \% \\
n=235(\%)\end{array}$ & $\begin{array}{l}\text { BMI loss } \geq 7 \% \\
n=39(\%)\end{array}$ & $P$ value \\
\hline \multicolumn{4}{|l|}{ Leucopenia } & \multicolumn{4}{|l|}{ Rash } \\
\hline Total & $190(58.5)$ & $26(66.7)$ & 0.33 & Total & $40(12.3)$ & $3(7.7)$ & 0.40 \\
\hline Grade $1-2$ & $176(54.2)$ & $25(64.1)$ & 0.24 & Grade $1-2$ & $36(11.1)$ & $1(2.6)$ & 0.13 \\
\hline Grade $3-4$ & $14(4.3)$ & $1(2.6)$ & 0.61 & Grade $3-4$ & $4(1.2)$ & $2(5.1)$ & 0.10 \\
\hline \multicolumn{4}{|c|}{ Thrombocytopenia } & \multicolumn{4}{|c|}{ Urination disorder } \\
\hline Total & $72(22.2)$ & $9(23.1)$ & 0.90 & Total & $10(3.1)$ & $1(2.6)$ & 0.86 \\
\hline Grade $1-2$ & $62(19.1)$ & $9(23.1)$ & 0.55 & Grade $1-2$ & $10(3.1)$ & $1(2.6)$ & 0.86 \\
\hline Grade 3-4 & $10(3.1)$ & 0 & 1 & Grade 3-4 & 0 & 0 & \\
\hline \multicolumn{4}{|c|}{ Nausea/vomiting } & \multicolumn{4}{|c|}{ Renal disorder } \\
\hline Total & $150(46.2)$ & $19(48.7)$ & 0.76 & Total & $2(0.6)$ & $2(5.1)$ & 0.033 \\
\hline Grade $1-2$ & $149(45.8)$ & $19(48.7)$ & 0.73 & Grade $1-2$ & $2(0.6)$ & $2(5.1)$ & 0.033 \\
\hline Grade 3-4 & $1(0.3)$ & 0 & 1 & Grade 3-4 & 0 & 0 & \\
\hline \multicolumn{4}{|l|}{ Diarrhea } & \multicolumn{4}{|c|}{ Hepatic disorder } \\
\hline Total & $102(31.4)$ & $19(48.7)$ & 0.033 & Total & $44(13.5)$ & 7 (17.9) & 0.46 \\
\hline Grade $1-2$ & $91(28.0)$ & $16(41.0)$ & 0.09 & Grade $1-2$ & $42(12.9)$ & 7 (17.9) & 0.39 \\
\hline Grade 3-4 & $11(3.4)$ & $3(7.7)$ & 0.20 & Grade 3-4 & $2(0.6)$ & 0 & 1 \\
\hline \multicolumn{4}{|c|}{ Hand-foot syndrome } & \multicolumn{4}{|l|}{ Neurotoxicity } \\
\hline Total & $225(69.2)$ & $27(69.2)$ & 1 & Total & $53(16.3)$ & $5(12.8)$ & 0.58 \\
\hline Grade $1-2$ & $216(66.5)$ & $25(64.1)$ & 0.77 & Grade $1-2$ & $53(16.3)$ & $5(12.8)$ & 0.58 \\
\hline Grade 3-4 & $9(2.8)$ & $2(5.1)$ & 0.42 & Grade $3-4$ & 0 & 0 & \\
\hline \multicolumn{8}{|c|}{ Radiation proctitis } \\
\hline Total & $198(60.9)$ & $24(61.5)$ & 0.94 & & & & \\
\hline Grade $1-2$ & $192(59.1)$ & $21(53.8)$ & 0.53 & & & & \\
\hline Grade $3-4$ & $6(1.8)$ & $3(7.7)$ & 0.041 & & & & \\
\hline
\end{tabular}

$B M I$ body mass index
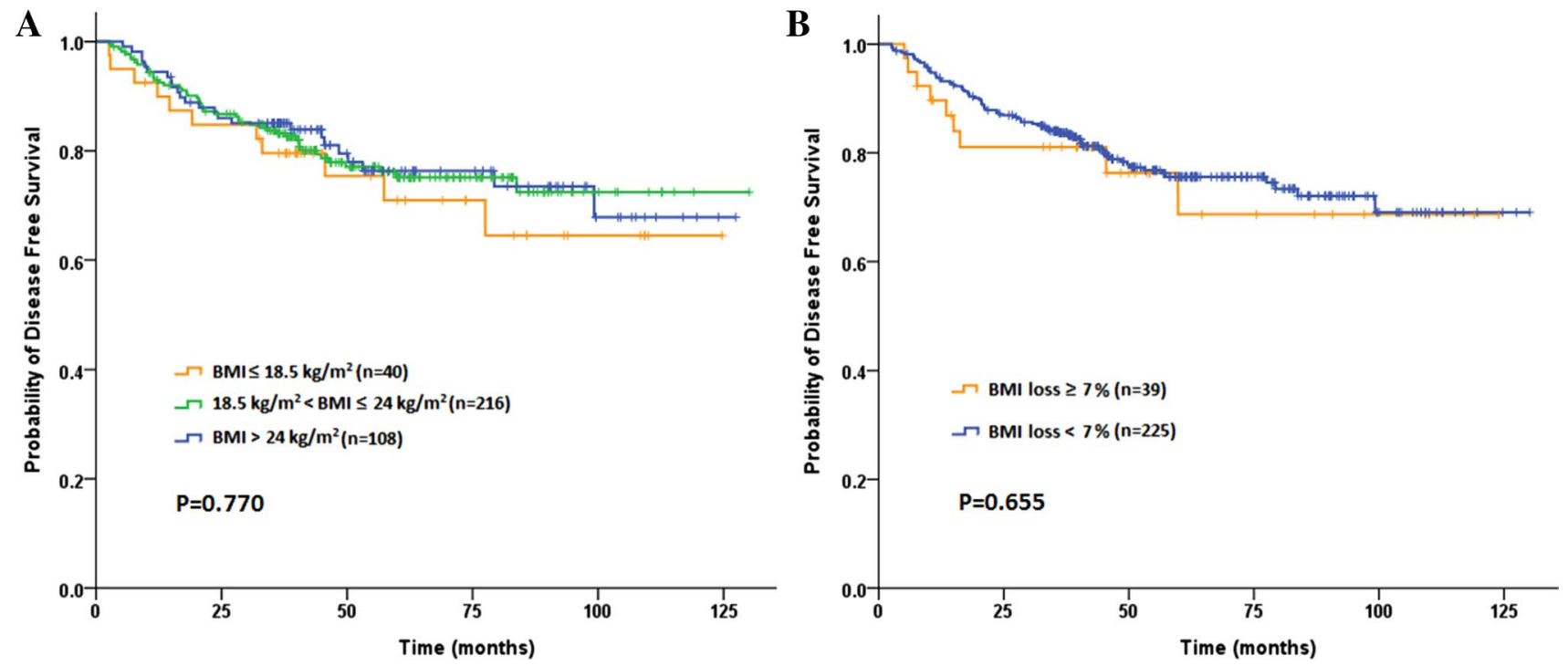

Fig. 2 Kaplan-Meier curve comparing 3-year disease-free survival (DFS) rate by a baseline BMI classification, b BMI loss during preoperative chemoradiotherapy inpatients with locally advanced rectal cancer 

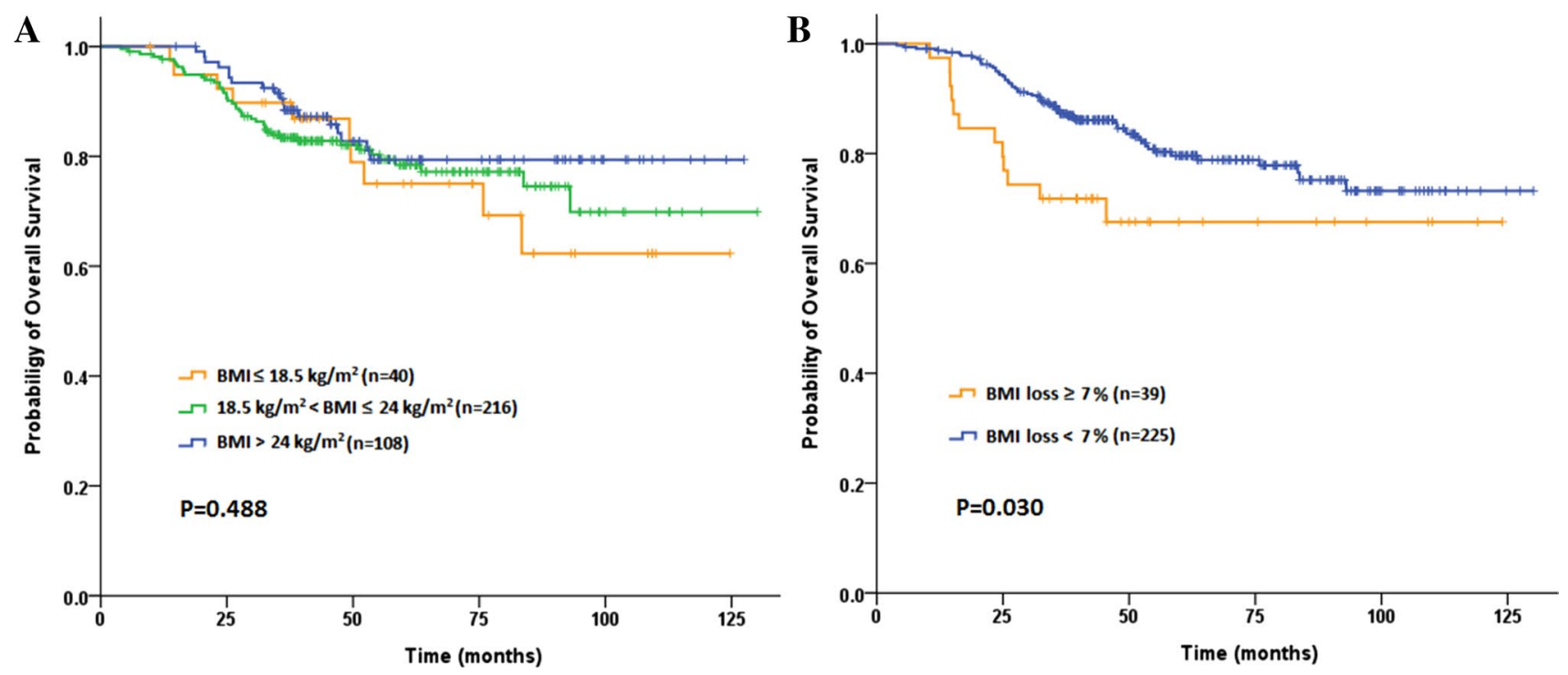

Fig. 3 Kaplan-Meier curve comparing 3-year overall survival (OS) rate by a baseline BMI classification, b BMI loss during preoperative chemoradiotherapy in patients with locally advanced rectal cancer

Table 5 Univariate and multivariate analysis of risk factors for overall survival in the patients with locally advanced rectal cancer

\begin{tabular}{|c|c|c|c|c|}
\hline \multirow[t]{2}{*}{ Variable } & \multicolumn{2}{|l|}{ Univariate } & \multicolumn{2}{|l|}{ Multivariate } \\
\hline & $\mathrm{HR}(95 \% \mathrm{CI})$ & $P$ value & $\mathrm{HR}(95 \% \mathrm{CI})$ & $P$ value \\
\hline Age $>60$ year & $1.360(0.856-2.162)$ & 0.19 & & \\
\hline Gender (female) & $1.139(0.702-1.847)$ & 0.60 & & \\
\hline PreCRT BMI $\leq 18.5 \mathrm{~kg} / \mathrm{m}^{2}$ & $1.248(0.640-2.436)$ & 0.52 & & \\
\hline PreCRT weight $\leq 50 \mathrm{~kg}$ & $1.430(0.784-2.607)$ & 0.24 & & \\
\hline cT stage 4 & $1.439(0.867-2.387)$ & 0.16 & & \\
\hline cTNM stage III & $1.269(0.734-2.193)$ & 0.39 & & \\
\hline $\mathrm{DAV} \leq 5 \mathrm{~cm}$ & $1.158(0.947-1.417)$ & 0.15 & & \\
\hline Tumor size $\geq 3 \mathrm{~cm}$ & $1.158(0.701-1.915)$ & 0.57 & & \\
\hline Histopathology (poor grade) & $1.175(0.689-2.002)$ & 0.55 & & \\
\hline Number of retrieved LNs $\geq 12$ & $0.776(0.456-1.322)$ & 0.35 & & \\
\hline ypT stage $3-4$ & $2.411(1.462-3.976)$ & 0.001 & $1.781(1.054-3.010)$ & 0.031 \\
\hline ypN stage $1-2$ & $3.227(2.031-5.126)$ & $<0.001$ & $2.571(1.576-4.192)$ & $<0.001$ \\
\hline Preoperative CRT with oxaliplatin & $0.466(0.202-1.075)$ & 0.07 & & \\
\hline Completed dose of preoperative chemotherapy $>50 \%$ & $0.470(0.253-0.874)$ & 0.017 & $0.532(0.284-0.997)$ & 0.049 \\
\hline Radiotherapy completion & $0.627(0.195-2.015)$ & 0.43 & & \\
\hline Anterior resection & $0.694(0.438-1.100)$ & 0.12 & & \\
\hline Acceptance of postoperative chemotherapy & $0.798(0.457-1.393)$ & 0.43 & & \\
\hline Duration of postoperative chemotherapy $\geq 12$ weeks & $0.843(0.489-1.453)$ & 0.45 & & \\
\hline $\mathrm{BMI}$ loss $\geq 7 \%$ during preoperative CRT & $1.961(1.055-3.647)$ & 0.030 & $1.984(1.061-3.709)$ & 0.032 \\
\hline
\end{tabular}

$B M I$ body mass index, $H R$ hazard ratio, $C I$ confidence interval, $D A V$ distance of inferior tumor margin from the anal verge, $c T$ stage clinical tumor stage, $c T N M$ stage clinical tumor-node-metastasis classification, LNs lymphonodus, ypT stage pathologic tumor stage after chemoradiotherapy, ypN stage pathologic node stage after chemoradiotherapy, $C R T$ chemoradiotherapy

to those parameters, and our data provided an essential clue for further research. Moreover, we failed to evaluate the impact of different body composition changes during CRT on the patient's oncologic outcome. Skeletal muscle and visceral adipose tissue loss might be the genuine factors contributing to poor survival according to the previous report (Dalal et al. 2012). Finally, 5-year survival outcome was unavailable due to insufficient follow-up time. 
This limitation may have led to underestimating the weight loss impact on long-term survival. Despite the limitations mentioned above, our study indeed raised the importance of emphasizing the nutritional status changes and nutrition support during neoadjuvant treatment for LARC patients.

\section{Conclusions}

Severe weight loss during preoperative CRT compromised the survival outcome of patients with LARC. Routine accurate monitoring of weight change, patient education and nutritional counseling, as well as proper supplement for nutritional balance during neoadjuvant treatment, are suggested as the integral part of the multidisciplinary approach for treating LARC patients.

Acknowledgments We would like to thank the all colleagues of Department of Colorectal Surgery and Radiation Oncology in Sun Yat-sen University Cancer Center, who have involved with performing the treatment for current study.

\section{Compliance with ethical standards}

Conflict of interest The authors declare that they have no competing interests.

Ethical approval All procedures performed in studies involving human participants were in accordance with the ethical standards of the institutional and/or national research committee and with the 1964 Helsinki Declaration and its later amendments or comparable ethical standards. A waiver of informed consent was requested, and the approval was obtained from independent ethics committees at Sun Yat-Sen University Cancer Center.

Open Access This article is distributed under the terms of the Creative Commons Attribution 4.0 International License (http://creativecommons.org/licenses/by/4.0/), which permits unrestricted use, distribution, and reproduction in any medium, provided you give appropriate credit to the original author(s) and the source, provide a link to the Creative Commons license, and indicate if changes were made.

\section{References}

Adachi T et al (2015) Lower body mass index predicts worse cancerspecific prognosis in octogenarians with colorectal cancer. J Gastroenterol. doi:10.1007/s00535-015-1147-z

Anandavadivelan P, Brismar TB, Nilsson M, Johar AM, Martin L (2016) Sarcopenic obesity: a probable risk factor for dose limiting toxicity during neo-adjuvant chemotherapy in oesophageal cancer patients. Clin Nutr 35:724-730. doi:10.1016/j.clnu.2015.05.011

Arrieta $\mathrm{O}$ et al (2010) Association of nutritional status and serum albumin levels with development of toxicity in patients with advanced non-small cell lung cancer treated with paclitaxelcisplatin chemotherapy: a prospective study. BMC Cancer 10:50. doi:10.1186/1471-2407-10-50
Arrieta O et al (2015) Nutritional status, body surface, and low lean body mass/body mass index are related to dose reduction and severe gastrointestinal toxicity induced by afatinib in patients with non-small cell lung cancer. Oncologist 20:967-974. doi:10.1634/theoncologist.2015-0058

Bachmann J et al (2015) Influence of an elevated nutrition risk score (NRS) on survival in patients following gastrectomy for gastric cancer. Med Oncol 32:204. doi:10.1007/s12032-015-0631-x

Bao PP, Cai H, Peng P, Gu K, Su Y, Shu XO, Zheng Y (2016) Body mass index and weight change in relation to triple-negative breast cancer survival. Cancer Causes Control 27:229-236. doi:10.1007/s10552-015-0700-7

Bosset JF et al (2006) Chemotherapy with preoperative radiotherapy in rectal cancer. N Eng J Med 355:1114-1123. doi:10.1056/ NEJMoa060829

Campbell PT, Newton CC, Dehal AN, Jacobs EJ, Patel AV, Gapstur SM (2012) Impact of body mass index on survival after colorectal cancer diagnosis: the Cancer Prevention Study-II Nutrition Cohort. J Clin Oncol 30:42-52. doi:10.1200/JCO.2011.38.0287

Cooper AB et al (2015) Characterization of anthropometric changes that occur during neoadjuvant therapy for potentially resectable pancreatic cancer. Ann Surg Oncol 22:2416-2423. doi:10.1245/ s10434-014-4285-2

Dalal S et al (2012) Relationships among body mass index, longitudinal body composition alterations, and survival in patients with locally advanced pancreatic cancer receiving chemoradiation: a pilot study. J Pain Symptom Manag 44:181-191. doi:10.1016/j. jpainsymman.2011.09.010

Das U, Patel S, Dave K, Bhansali R (2014) Assessment of nutritional status of gynecological cancer cases in India and comparison of subjective and objective nutrition assessment parameters. South Asian J Cancer 3:38-42. doi:10.4103/2278-330X.126518

Diaz-Gonzalez JA et al (2006) Prognostic factors for disease-free survival in patients with $\mathrm{T} 3-4$ or $\mathrm{N}+$ rectal cancer treated with preoperative chemoradiation therapy, surgery, and intraoperative irradiation. Int J Radiat Oncol 64:1122-1128. doi:10.1016/j. ijrobp.2005.09.020

Fontana L, Eagon JC, Colonna M, Klein S (2007) Impaired mononuclear cell immune function in extreme obesity is corrected by weight loss. Rejuv Res 10:41-46. doi:10.1089/rej.2006.0504

Gudny Geirsdottir O, Thorsdottir I (2008) Nutritional status of cancer patients in chemotherapy; dietary intake, nitrogen balance and screening. Food Nutr Res. doi:10.3402/fnr.v52i0.1856

Hede P, Sorensson MA, Polleryd P, Persson K, Hallgren T (2015) Influence of BMI on short-term surgical outcome after colorectal cancer surgery: a study based on the Swedish national quality registry. Int J Colorectal Dis 30:1201-1207. doi:10.1007/ s00384-015-2280-0

Iseki $Y$ et al (2015) Impact of the preoperative controlling nutritional status (CONUT) score on the survival after curative surgery for colorectal cancer. PLoS ONE 10:e0132488. doi:10.1371/journal. pone. 0132488

Kong M, Hong SE, Choi WS, Kim SY, Choi J (2012) Preoperative concurrent chemoradiotherapy for locally advanced rectal cancer: treatment outcomes and analysis of prognostic factors. Cancer Res Treat 44:104-112. doi:10.4143/crt.2012.44.2.104

Koom WS et al (2012) Nutritional status of patients treated with radiotherapy as determined by subjective global assessment. Radiat Oncol J 30:132-139. doi:10.3857/roj.2012.30.3.132

Mandard AM et al (1994) Pathologic assessment of tumor regression after preoperative chemoradiotherapy of esophageal carcinoma. Clinicopathologic correlations. Cancer 73:2680-2686

Martin L, Lagergren P (2009) Long-term weight change after oesophageal cancer surgery. Br J Surg 96:1308-1314. doi:10.1002/ bjs. 6723 
Matsuo K, Moeini A, Cahoon SS, Machida H, Ciccone MA, Grubbs BH, Muderspach LI (2016) Weight change pattern and survival outcome of women with endometrial cancer. Ann Surg Oncol. doi:10.1245/s10434-016-5237-9

Miyata $\mathrm{H}$ et al (2012) Randomized study of clinical effect of enteral nutrition support during neoadjuvant chemotherapy on chemotherapy-related toxicity in patients with esophageal cancer. Clin Nutr 31:330-336. doi:10.1016/j.clnu.2011.11.002

Parr CL et al (2010) Body-mass index and cancer mortality in the Asia-Pacific Cohort Studies Collaboration: pooled analyses of 424,519 participants. Lancet Oncol 11:741-752. doi:10.1016/ S1470-2045(10)70141-8

Qiu M et al (2015) Nutrition support can bring survival benefit to high nutrition risk gastric cancer patients who received chemotherapy. Support Care Cancer 23:1933-1939. doi:10.1007/ s00520-014-2523-6

Ravasco P, Monteiro-Grillo I, Camilo M (2012) Individualized nutrition intervention is of major benefit to colorectal cancer patients: long-term follow-up of a randomized controlled trial of nutritional therapy. Am J Clin Nutr 96:1346-1353. doi:10.3945/ ajcn.111.018838

Read JA, Choy ST, Beale PJ, Clarke SJ (2006) Evaluation of nutritional and inflammatory status of advanced colorectal cancer patients and its correlation with survival. Nutr Cancer 55:78-85. doi:10.1207/s15327914nc5501_10

Reeves GK, Pirie K, Beral V, Green J, Spencer E, Bull D, Million Women Study Collaboration (2007) Cancer incidence and mortality in relation to body mass index in the Million Women Study: cohort study. BMJ 335:1134. doi:10.1136/bmj.39367.495995.AE

Sakurai K et al (2016) Predictive potential of preoperative nutritional status in long-term outcome projections for patients with gastric cancer. Ann Surg Oncol 23:525-533. doi:10.1245/ s10434-015-4814-7

Sanchez-Lara K, Ugalde-Morales E, Motola-Kuba D, Green D (2013) Gastrointestinal symptoms and weight loss in cancer patients receiving chemotherapy. Br J Nutr 109:894-897. doi:10.1017/ S0007114512002073

Sauer R et al (2004) Preoperative versus postoperative chemoradiotherapy for rectal cancer. N Engl J Med 351:1731-1740. doi:10.1056/NEJMoa040694

Song KS et al (2011) Omega-3-polyunsaturated fatty acids suppress pancreatic cancer cell growth in vitro and in vivo via downregulation of Wnt/Beta-catenin signaling. Pancreatology 11:574-584. doi:10.1159/000334468

Uratani R et al (2015) Preoperative lower body mass index correlates with poorer prognosis in patients undergoing curative laparoscopic surgery for colorectal cancer. Anticancer Res 35:5639-5648

Valdes-Ramos R, Benitez-Arciniega AD (2007) Nutrition and immunity in cancer. Brit J Nutr 98(Suppl 1):S127-S132. doi:10.1017/ S0007114507833009

Xu Y, Qian SY (2014) Anti-cancer activities of omega-6 polyunsaturated fatty acids. Biomed J 37:112-119. doi: $10.4103 / 2319-4170.131378$

Yaqoob P, Calder PC (2007) Fatty acids and immune function: new insights into mechanisms. Br J Nutr 98(Suppl 1):S41-S45. doi:10.1017/S0007114507832995

Zeng Q et al (2014) Optimal cut-off values of BMI, waist circumference and waist:height ratio for defining obesity in Chinese adults. Br J Nutr 112:1735-1744. doi:10.1017/S0007114514002657 